\title{
Network complexity as a measure of information processing across resting-state networks: evidence from the Human Connectome Project
}

\author{
Ian M. McDonough * and Kaoru Nashiro \\ Center for Vital Longevity, School of Behavioral and Brain Sciences, University of Texas at Dallas, Dallas, TX, USA
}

Edited by:

Srikantan S. Nagarajan, University of

California, San Francisco, USA

\section{Reviewed by:}

Catie Chang, National Institutes of Health, USA

Krzysztof Gorgolewski, Max Planck Institute for Human Cognitive and Brain Sciences, Germany

*Correspondence:

lan M. McDonough, Center for Vital Longevity, University of Texas at

Dallas, 1600 Viceroy Dr. \#800,

Dallas, TX 75235, USA

e-mail: ian.mcdonough@gmail.com
An emerging field of research focused on fluctuations in brain signals has provided evidence that the complexity of those signals, as measured by entropy, conveys important information about network dynamics (e.g., local and distributed processing). While much research has focused on how neural complexity differs in populations with different age groups or clinical disorders, substantially less research has focused on the basic understanding of neural complexity in populations with young and healthy brain states. The present study used resting-state fMRI data from the Human Connectome Project (Van Essen et al., 2013) to test the extent that neural complexity in the BOLD signal, as measured by multiscale entropy (1) would differ from random noise, (2) would differ between four major resting-state networks previously associated with higher-order cognition, and (3) would be associated with the strength and extent of functional connectivity-a complementary method of estimating information processing. We found that complexity in the BOLD signal exhibited different patterns of complexity from white, pink, and red noise and that neural complexity was differentially expressed between resting-state networks, including the default mode, cingulo-opercular, left and right frontoparietal networks. Lastly, neural complexity across all networks was negatively associated with functional connectivity at fine scales, but was positively associated with functional connectivity at coarse scales. The present study is the first to characterize neural complexity in BOLD signals at a high temporal resolution and across different networks and might help clarify the inconsistencies between neural complexity and functional connectivity, thus informing the mechanisms underlying neural complexity.

\section{Keywords: functional connectivity, Human Connectome Project, information processing, multiscale entropy,} neural complexity, resting-state networks

\section{INTRODUCTION}

Research over the past decade has provided evidence that the temporal fluctuations of brain activity are crucial and fundamental properties of the human brain (e.g., Biswal et al., 2010; Deco et al., 2011; Wig et al., 2011). The synchrony, or correlation, of these temporal fluctuations between regions (i.e., functional connectivity) is one way to assess the communication of information in the brain and give rise to separate functional networks. These networks have been associated with specific cognitive processes (Seeley et al., 2007; Dosenbach et al., 2008; Vincent et al., 2008; Smith et al., 2009; Laird et al., 2011) and disruptions of these networks are associated with corresponding clinical disorders (e.g., Seeley et al., 2009; Posner et al., 2014). However, by concentrating efforts on understanding the correlations of temporal fluctuations, this research has largely ignored the pattern of these fluctuations within a region or network. Advances in signal processing methods have enabled the quantification of temporal patterns by assessing their randomness, or complexity (Costa et al., 2005). The more complex the pattern of brain activity, the more rich the information (e.g., Tononi et al., 1994, 1998; Garrett et al., 2013; Nakagawa et al., 2013) or more integrated the information (e.g., Vakorin et al., 2011; McIntosh et al., 2013) is within a network. Thus, whereas complex systems signify a normal and healthy state, highly regular (less complex) systems often mark dysfunction and disease (e.g., Pincus and Goldberger, 1994; Goldberger et al., 2002; Yang and Tsai, 2013). Consistent with this idea, measures of complexity have provided a useful tool for differentiating people of different age groups (e.g., McIntosh et al., 2008, 2013; Vakorin et al., 2011; Yang et al., 2012; Smith et al., 2013) and with different clinical disorders including Alzheimer's disease (e.g., Escudero et al., 2006; Mizuno et al., 2010; Yang et al., 2013b), autism (e.g., Bosl et al., 2011; Catarino et al., 2011; Ghanbari et al., 2013), attention deficit hyperactivity disorder (Gomez et al., 2013; Sokunbi et al., 2013), depression (e.g., Méndez et al., 2012), schizophrenia (Takahashi et al., 2010), tramatic brain injury (Beharelle et al., 2012), among others. As illustrated above, research is quickly demonstrating the utility of neural complexity as a marker of health, but our understanding is limited on how neural complexity is related to information processing and how it is characterized in a young, healthy system.

\section{MEASURING NEURAL COMPLEXITY}

Neural complexity is most often assessed using measures of entropy-the proportion of ordered patterns that can be 
detected in a signal (Pincus and Goldberger, 1994; Richman and Moorman, 2000; Lake et al., 2002). The fewer patterns that can be found within a signal (i.e., the more random the time series), the greater the signal complexity is. It has also been shown that recurring patterns within a physiological signal occur across a range of time scales (high to low frequencies) and so estimation of multiple time scales is necessary (Costa et al., 2005). Multiscale entropy (MSE) was developed to capture short-range/high frequency temporal complexity (i.e., fine scales) and long-range/low-frequency temporal complexity (i.e., coarse scales). Different time scales are calculated by down-sampling the original time series by averaging neighboring data points within non-overlapping windows. For a scale of 1 , the original time series would remain intact. For a scale of 2 , adjoining time points would be averaged, resulting in a time series half as long. It should be noted that the physiological nature of fine and coarse time scales remains unclear, but are thought to relate to different pathophysiological mechanisms (Mizuno et al., 2010; Yang et al., 2013b). One possibility is that fine time scales represent local information processing, while coarse time scales represent distributed information processing (e.g., Mizuno et al., 2010; Vakorin et al., 2011; McIntosh et al., 2013).

By using different time scales, MSE analysis can differentiate complex signals (assumed to carry meaningful information) from random noise (assumed to be unimportant). An increase in randomness of a signal does not always correspond to an increase in complexity because signals dominated by random fluctuations (e.g., white noise) are also highly irregular and should maximize entropy at fine time scales. However, completely random fluctuations should be characterized by decreases in complexity as time scales become coarser because these random fluctuations are smoothed out. In contrast, random fluctuations that carry $1 / f^{\beta}$ spectral properties (e.g., pink or red noise) should exhibit a constant level of complexity across time scales due to their fractal properties (e.g., Costa et al., 2005; Smith et al., 2013). Hence, complexity represents an intermediate pattern of order and randomness that is only fully captured when measured across multiple time scales. Indeed, MSE analyses have shown a distinct pattern of complexity across time scales and this pattern differs from both white noise (rapid decline in complexity) and pink noise (constant level of complexity). Using EEG and MEG, neural complexity at fine time scales is characterized by relatively low complexity. As time scales become coarser, neural complexity increases and often plateaus or dips at the coarsest scales (i.e., a skewed inverted-U pattern). While fewer studies have investigated neural complexity of the BOLD signal, two recent fMRI studies have shown decreasing levels of complexity as time scale increases (using 5-10 total scales rather than 20-40 total scales; Yang et al., 2012; Smith et al., 2013). Thus, the pattern of neural complexity across time scales differs depending on the imaging modality used (EEG/MEG or fMRI), which in turn makes it difficult to compare findings across studies.

\section{NEURAL COMPLEXITY: THEORIES AND EVIDENCE}

While it is widely agreed that neural complexity is related to information processing, different theories have been proposed to explain how the two are related (for a recent review, see Garrett et al., 2013). A few of these theories are briefly summarized below.

\section{A dynamic range of microstates}

Neural complexity might represent the range or capacity of the brain to explore alternative brain states. Different resting-state models have proposed that fluctuations in brain activity operate in a balanced state of stable and synchronous patterns of activity (Honey et al., 2007, 2009; Ghosh et al., 2008; Deco et al., 2011; Shew et al., 2009, 2011; McIntosh et al., 2010). Nodes within a network transiently synchronize into sets of co-activated brain regions and noise within a system helps propel the brain into these different states. These ideas are consistent with proposals made by Friston et al. (2012), who argue that a characteristic feature of the brain is its tendency to wander, or not settle in to any particular state. By retaining an optimal degree of instability, a brain system can explore alternative hypotheses about the causes of incoming stimuli and permits the brain to learn by discovery (van Leeuwen, 2008). These wandering dynamics allow the system to converge on optimal responses to environmental demands. Admittedly, these ideas remain abstract, but the idea is that systems engaging in greater transition or exploration between different states (e.g., stable and synchronous states) have a greater propensity for information processing, thus increasing the level of complexity in a system.

\section{Facilitation of neuronal firing}

A more basic proposal is that the randomness of fluctuating brain activity represents a moderate level of noise in a system that enhances the probability of neuronal firing. Stochastic resonance models have illustrated that adding noise in a neural system can help subthreshold neurons reach firing thresholds (e.g., Wiesenfeld and Moss, 1995; Faisal et al., 2008; McDonnell and Ward, 2011). Moderate amounts of noise would lead to increased neuronal firing and, therefore, the potential for more information processing. Of course, too much noise would result in too much firing and a saturation of the signal. However, in this context there seems to be a balancing point where deviations from a moderate level of randomness (i.e., in a healthy system) could lead to abnormal firing rates, and potentially clinical disorders.

\section{Regulation of neural synchrony}

Rather than simply aiding neuronal firing, another proposal is that the degree of randomness in a system can facilitate or even inhibit the likelihood of synchrony between brain regions. Ghanbari et al. (2013) argued that a tightly regulated system is composed of brain activity low in complexity (i.e., predictable temporal patterns) possibly regulated by successful excitation and inhibition of glutamate and gamma-aminobutyric acid (GABA) neurotransmitters. More predictable signals (less neural complexity) establish an environment that facilitates phase relationships between brain regions, thus increasing the probability of synchrony, and in turn, information exchange across distributed brain regions. In contrast, more random signals (greater neural complexity) establish an environment in which phase relationships are difficult to obtain, thus decreasing the probability of synchrony and the amount of information exchanged across brain regions. Unlike previous proposals, this proposal suggests an inverse relationship with the degree of neural complexity and information processing. To the extent that different time scales are 
associated with different levels of complexity, the degree of synchrony across brain regions should differ between fine and coarse time scales.

Relatedly, neural models of information processing have suggested that both the degree of synchrony and time scale determine the maximum information transfer between neurons (Baptista and Kurths, 2008). Specifically, information processing should be maximized when neurons synchronize at coarse time scales (i.e., lower frequencies), but desynchronize at fine time scales (i.e., higher frequencies). Moreover, it has been suggested that coarse time scales reflect long-range interactions across distributed neural populations, while fine time scales reflect interconnectivity among local neural populations (Mizuno et al., 2010; Vakorin et al., 2011; McIntosh et al., 2013). Thus, the combination of these ideas would predict (1) less neural complexity is associated with greater synchrony between brain regions and (2) fine and coarse time scales should exhibit an inverse relationship (i.e., fine time scales should be characterized by low synchrony and greater information processing, and vice versa for coarse time scales).

\section{Evidence from functional connectivity}

The theories reviewed above all suggest that neural complexity is, in some way, related to neuronal dynamics that can either facilitate or inhibit patterns of neuronal firing, thus promoting information communication within local or across distributed neural populations. To the extent that these potential mechanisms facilitate information communication, neural complexity should be related to other established forms of information communication such as functional connectivity (see section Introduction). It could be argued that the first two theories (range of microstates and facilitation of neuronal firing) would predict a positive relationship between neural complexity and functional connectivity (i.e., greater neural complexity should be associated with greater functional connectivity). In regards to the last theory of neural synchrony, Ghanbari et al. (2013) make specific predictions of an inverse relationship between neural complexity and functional connectivity, while other researchers generally suggest that neural complexity should be positively associated with functional connectivity (e.g., Vakorin et al., 2011; McIntosh et al., 2013).

Evidence from EEG and MEG studies are able to capture a wide range of time scales to test (1) the extent that there is a relationship between neural complexity and connectivity, (2) the direction of this potential relationship, and (3) whether these effects differ at fine and coarse time scales. One of the first empirical demonstrations that neural complexity and functional connectivity are related was conducted by Mišić et al. (2011). Using graph theory metrics, they showed that the centrality of each node was positively associated with neural complexity at all-time scales. Centrality was measured by node degree (number of connections), regional efficiency (ease of accessibility from other nodes), and betweenness (the tendency to be in between other nodes). Interestingly, they found that this positive relationship was weaker at fine time scales, consistent with the idea that fine scales are dominated by more random signals, which decrease the likelihood of synchronization (Ghanbari et al., 2013). Consistent with these findings, McIntosh et al. (2013) investigated age differences in entropy and functional connectivity using both EEG and MEG.
At fine scales, they found age-related increases in both complexity and within-hemisphere (i.e., local) connectivity. At coarse scales, the reverse occurred; they found age-related decreases in both complexity and across-hemisphere (distributed) connectivity.

However, Ghanbari et al. (2013) found different complexityconnectivity relationships when using MEG and children with autism spectrum disorders (ASD) compared with typically developing controls. In the frontal lobe/alpha-band signal, they found ASD-related decreases in complexity, but ASD-related increases in functional connectivity. In the temporal lobe/broad-band signal, they found ASD-related increases complexity, but ASD-related decreases in functional connectivity. These inverse relationships were similar across many different time scales and scalp electrodes in their study. Only one study fMRI study has investigated both neural complexity and functional connectivity within the same study (Yang et al., 2013a). This study investigated the effects of the gene Apolipoprotein E (APOE), a risk factor for Alzheimer's disease, on BOLD signals. They found that older adults with the APOE E4 gene had reduced neural complexity in the posterior cingulate and increased functional connectivity from this region to frontal regions. Because of the limitation of the sampling rate and length of fMRI time series, this effect can only be attributed to fine time scales. Note that in this study, the neural complexity and connectivity were never directly compared. Nevertheless, these latter two studies implicate an inverse relationship between neural complexity and functional connectivity.

\section{LIMITATIONS OF PRIOR STUDIES}

While complexity and connectivity are often correlated, the directions of these effects are not consistent. One issue is that the majority of studies investigating complexity-connectivity relationships have used populations at different ages or with clinical disorders rather than a population with a healthy and functional system. This method is problematic because the complexity-connectivity relationship could also be different in these populations. For instance, complexity-connectivity relationships could be positive in young adults, but negative in older adults. Additionally, these group differences might selectively impact fine or coarse time scales, further complicating interpretations.

Comparisons across studies also are made difficult by the different modalities used, which in turn affect the precision of the parameters used to calculate complexity, namely the sampling rate and length of the time series. For example, EEG and MEG have both shorter sampling rates and often have longer time series, thus enabling a more precise measure of both fine and coarse time scales. In contrast, the longer sampling rate and short time series in fMRI allow for only a few reliable time scales (at most) to be included in the analysis. The observed decrease in complexity across the time scales in fMRI suggests that complexity values at fine scales using fMRI might be similar to complexity values at mid or coarse scales using EEG or MEG. But of course, fMRI allows for a more precise localization of brain activity, thus being able to tie neural complexity to cognitive functions known to subserve specific brain regions or networks.

Lastly, few studies have attempted to test the degree that neural complexity differs between networks. Different resting-state 
networks (RSNs) are thought to represent large-scale component processes involved in different aspects of cognition (e.g., Seeley et al., 2007; Dosenbach et al., 2008; Vincent et al., 2008; Smith et al., 2009; Laird et al., 2011). Interestingly, because functional networks are often defined by the similarity of their time series, each brain region within a network should have the same pattern of neural complexity, but the level of complexity in each network might still differ from one another. These inter-network complexity differences might provide insight into the dynamics of each network including differences in local and distributed information processing.

\section{THE PRESENT STUDY}

We addressed the limitations in prior studies in four ways. First, we aimed to capture a larger range of MSE in fMRI than has been used in previous studies by utilizing an fMRI time series with a very short sampling rate and long epochs within a scanning session. Specifically, data was used from the Human Connectome Project (HCP; Van Essen et al., 2013) in a set of 20 healthy young adults. The resting-state data from HCP consists of four resting state scans of 1200 time points each with a sampling rate of $720 \mathrm{~ms}$. Not only will this sampling rate enable us to test whether patterns of MSE match those predicted by model simulations (e.g., Nakagawa et al., 2013), but this dataset also might allow a better comparison with studies investigating MSE in EEG/MEG, with the caveat that the slow hemodynamic response as measured via BOLD fMRI is fundamentally limited in temporal resolution. We predicted that a skewed inverted-U pattern would be found in neural complexity across time scales-the first fMRI study to show similar patterns of neural complexity patterns often found in EEG/MEG (e.g., Mišić et al., 2011; McIntosh et al., 2013; Yang et al., 2013b) and BOLD simulations (e.g., Nakagawa et al., 2013).

Second, we aimed to distinguish patterns of neural complexity from white, pink, and red noise. Few studies have attempted to estimate complexity from simulated noise and compare them to complexity values in BOLD signals. To the extent that each time scale has a complexity value that differs between the BOLD signal and simulated noise, those time scales are more likely to contain information related to neural signals, potentially subserving information processing. Additionally, simulating noise can address the null hypothesis that the pattern of complexity in BOLD signals differs from the pattern of complexity found in noise. While one study showed evidence that neural complexity in BOLD signals did not differ from white noise or from BOLD signals in white matter at fine time scales (Smith et al., 2013), we predicted that the higher sampling rate would allow for a better distinction between random noise (including white, pink, and red noise) and neural complexity in BOLD signals. In addition, we included BOLD signals from cerebral spinal fluid (CSF) as an additional test that neural complexity in gray matter differs from complexity in physiological signals presumably not neural in origin.

Third, using fMRI allows the characterization of neural complexity across different RSNs, which we refer to as network complexity. Network complexity was assessed in four key RSNs (default mode, cingulo-opercular, left and right frontoparietal), each of which has been implicated in higher-order cognition.
For example, the default mode network (DMN) has been associated with a variety of cognitive domains including episodic memory, working memory, and self-reflection (e.g., Hampson et al., 2006; Buckner et al., 2008; Spreng et al., 2009). The cingulo-opercular network (CON) has been associated with the orientation/maintenance of attention and has been correlated with processing speed (e.g., Dosenbach et al., 2008; Menon and Uddin, 2010; Touroutoglou et al., 2012). The left and right frontoparietal networks (LFN and RFN) have been associated with the control of working memory, and decision making during goaldirected actions (e.g., Seeley et al., 2007; Dosenbach et al., 2008; Vincent et al., 2008). To the extent that information communication differs between RSNs, we predict that network complexity also would differ between RSNs at both fine and coarse time scales.

Lastly, the current study examined the relationship between neural complexity and functional connectivity in each of the RSNs to test our hypothesis that they would be related in all networks, but the complexity-connectivity relationship would depend on time scale. Critically, these analyses were conducted in a group of healthy young adults, thus providing a baseline from which to interpret other findings using populations of different ages or clinical disorders. Only one other fMRI study, to our knowledge, has assessed both complexity and connectivity in the same study, but no direct comparisons between the two measures were made, leaving open the question of how individual differences in the two measures might be expressed (Yang et al., 2013a).

\section{MATERIALS AND METHODS PARTICIPANTS}

The first available 20 participants (22-35 years of age; 12 females) provided by the HCP (WU-Minn Consortium) were used in the present study. The HCP is a long-term study enabling the exploration of human brain circuits. These participants were unrelated to each other, relatively healthy individuals that were free of a prior history of significant psychiatric or neurological illnesses, but could have a history of smoking, heavy drinking, or recreational drug use without having experienced severe symptoms. All participants gave informed consent as approved by the Washington University in St. Louis institutional review board.

\section{fMRI ACQUISITION}

All data were acquired on a Siemens Skyra 3T scanner housed at Washington University in St. Louis. The scanner had a customized SC72 gradient insert and a customized body transmitter coil with $56 \mathrm{~cm}$ bore size (diffusion: $\mathrm{Gmax}=100 \mathrm{mT} / \mathrm{m}$, max slew rate $=$ $91 \mathrm{mT} / \mathrm{m} / \mathrm{ms}$; readout/imaging: Gmax $=42 \mathrm{mT} / \mathrm{m}$, $\max$ slew rate $=200 \mathrm{mT} / \mathrm{m} / \mathrm{ms}$ ). The HCP Skyra had the standard set of Siemen's shim coils (up to 2nd order) and used Siemen's standard 32 channel head coil. BOLD fMRI data were acquired using a T2* weighted gradient-echo EPI sequence with 72 axial slices per volume, $104 \times 90$ matrix $\left(2.0 \times 2.0 \times 2.0 \mathrm{~mm}^{3}\right)$, FOV $=208 \mathrm{~mm}$, $\mathrm{TE}=33.1 \mathrm{~ms}, \mathrm{TR}=720 \mathrm{~ms}, \mathrm{FA}=52^{\circ}$. Across four scanning sessions, a total of 4800 frames were acquired.

Visual stimuli (i.e., a cross-hair) were presented and participant responses were collected using a Dell Optiplex 790 computer, 
running an Intel Core i3-2100 with $8 \mathrm{~GB}$ of RAM and 64-bit Windows 7 Enterprise SP1. The E-Prime version was E-Prime 2.0 Professional Production Release (2.0.10.242). Visual stimuli were projected with a NEC V260X projector onto a lucite screen at $1024 \times 768$ resolution, and viewed by the participant using a mirror mounted on the top of the head coil. Participant responses were registered on a customized fiber-optic button box.

\section{RESTING-STATE fMRI PROCEDURE}

Scans from resting-state fMRI data were acquired in four scanning sessions of approximately $15 \mathrm{~min}$ each with eyes open relaxed and fixated on a projected bright cross-hair on a dark background presented in a darkened room. Across sessions, oblique axial acquisitions alternated between phase encoding in a right-to-left direction and phase encoding in a left-to-right direction.

\section{fMRI PREPROCESSING}

Postprocessed fMRI datasets were used in the present study, which consisted of standard processing methods using FSL (Jenkinson et al., 2002, 2012; http://fsl.fmrib.ox.ac.uk/fsl/fslwiki/). Below briefly summarizes the HCP processing pipeline (Glasser et al., 2013). First, gradient-non-linearity-induced distortion was corrected for all images. Next, FMRIB's Linear Image Registration Tool (FLIRT) was used for motion correction using the singleband reference (SBRef) image as the target. The FSL toolbox "topup" (Andersson et al., 2003) was used to estimate the distortion field in the functional images. The SBRef image was used for EPI distortion correction and is registered to the T1w image. One-step spline resampling from the original EPI frames to atlas space was applied to all transforms. Lastly, image intensity was normalized to mean of 10000 and bias field was removed. Additional processing steps were used to reduce variance unlikely to reflect neuronal activity using the REST toolbox (Song et al., 2011). These steps included removing linear trends and regression on six motion correction parameters and average BOLD signal using three masks included in the REST toolbox, which included a whole brain mask to remove global mean signal ${ }^{1}$.

\section{DATA ANALYSIS}

\section{Resting-state fMRI analysis}

Dual regression analyses (Beckmann et al., 2009; Filippini et al., 2009) were implemented to isolate the time series within four major RSNs associated with higher-order cognition and to estimate subject-specific functional connectivity within each of

\footnotetext{
${ }^{1}$ Global signal regression is controversial because doing so can sometimes remove signal of interest, increase spurious long-range connections, and create "anti-correlated" networks (e.g., Murphy et al., 2009; Saad et al., 2012). However, removing global signal regression has also been shown to remove artifactual correlations due to movement (e.g., Satterthwaite et al., 2013). While more research needs to be conducted to verify instances when global signal removal is appropriate, the goals of the present study were to capture a time series representative of large-scale brain networks, while reducing the contribution of movement-related noise to these estimates. However, systematic investigations regarding the impact of removing global signal on MSE estimates are necessary to fully understand its impact on these MSE estimates.
}

the networks. Using this technique has several advantages over seed-based methods of identifying RSNs. Specifically, this method can help minimize the contribution of physiological-related signal to the resting-state network components (e.g., Kiviniemi et al., 2003; Beckmann and Smith, 2004), is much more robust to motion than seed-based methods (Calhoun and Adali, 2012), and a single time series can be extracted that is common across all voxels to be used to estimate network complexity.

In the dual regression analysis, we used four a priori templates from Smith et al. (2009) to reconstruct subject-specific time series and spatial maps for DMN (component 4), CON (component 8), LFN (component 10), and RFN (component 9). The first regression model used each template as a spatial predictor for the participant's $4 \mathrm{D}$ data, producing a set of individual regression weights in the time domain (i.e., a time series for each spatial map). Using this time series as a temporal predictor for the $4 \mathrm{D}$ BOLD data, the second regression equation estimated the individual regression weights in the spatial domain. These regression weights represented the degree that the time series in each voxel matched the time series for that component, and thus can be interpreted as a measure of functional-connectivity strength (e.g., Calhoun and Adali, 2012). Due to computational constraints, dual regression analyses were applied separately for each of the four scanning sessions instead of concatenating the four scanning sessions prior to computing the dual regressions.

The resulting subject-specific time series for each network of interest was used to calculate the network complexity of each RSN using multiscale entropy (see section Multiscale Entropy (MSE) Analysis) and averaged across the four scanning sessions. The resulting subject-specific spatial maps were Z-transformed and thresholded at $Z>3.29$ ( $p \leq 0.001$ ). The strength of functional connectivity was estimated by averaging the thresholded $Z$-values within each network for each subject. The spatial extent of functional connectivity was estimated by counting the number of significant voxels within each network for each subject. Data was mapped onto the cortical surface using CARET software (Van Essen et al., 2001).

\section{MultiScale Entropy (MSE) analysis}

After the time series from each RSN was extracted, the complexity of each network was estimated using MSE developed by Costa et al. (2005), which estimates sample entropy at different time scales. First, fine to more coarse-grained time series were created by down-sampling the original time series (i.e., averaging neighboring data points within non-overlapping windows). Second, sample entropy was estimated for the time series at each time scale (1-25 scales). Sample entropy is defined as the natural logarithm of the conditional probability that a given pattern of data of a specified length $(m)$ repeats at the next time point for the entire time series at a given scale factor (of a dataset with a total length $N$ ). It considers subsequent patterns to be a repeat of the given pattern if they match within a certain tolerance $(r)$ such that larger tolerance values increase the number of matches (Richman and Moorman, 2000; Lake et al., 2002). To the extent that a time series has a greater number of pattern matches, the time series is less random, and the entropy value is lower. In contrast, a smaller number of pattern matches is characterized as 
being more random, yielding a greater entropy value. We selected our parameters based on those used in prior studies investigating MSE using fMRI, $m=2$ and $r=0.5$ (e.g., Smith et al., 2013; Sokunbi et al., 2013). While exact parameters sometimes differ across studies, one of the advantages of using sample entropy (as used in MSE analysis) over other estimates of entropy (e.g., approximate entropy; Pincus, 1991) is that sample entropy is consistent over a broad range of possible $r, M$, and $N$ values (e.g., Richman and Moorman, 2000; Lake et al., 2002; Sokunbi et al., 2013) $)^{2}$.

\section{Simulations of neural complexity in noise and in CSF}

Three profiles of noise were created and complexity in these noise profiles was compared with network complexity in each RSN. Patterns of complexity within brain signals should be different than those found in noise, potentially representing meaningful information processing. Time series consisting of white, pink, and red noise were estimated using f_alpha scripts (http://people.sc.fsu.edu/ jburkardt/m_src/ colored_noise/colored_noise.html). White noise is a completely unpredictable signal with a constant power spectral density. Pink noise ( $1 / f$ noise) and red noise $\left(1 / f^{2}\right)$ are signals with a power spectral density that is inversely proportional to the frequency. Some evidence has suggested that the BOLD signal contains $1 / f$ and $1 / f^{2}$ spectral properties (e.g., Zarahn et al., 1997; Bullmore et al., 2004; Milstein et al., 2008; He et al., 2010; He, 2011) that are indicative of time points being temporally autocorrelated with more "noise" at some frequencies than others with this temporal autocorrelation potentially being time-lagged. Of course, the assumption is that some aspects of noise are not meaningful to information processing, even if the noise is structured. Three sets of simulated time series (white, pink, and red noise) were generated to match the length and variance of the time series of each participant for each network, totaling 640 time series (20 participants $x$ four sessions $x$ four networks $x$ three types of noise). MSE analyses were then applied to each of the time series and averaged across the sessions and networks ${ }^{3}$.

\footnotetext{
${ }^{2}$ We should note that two studies (Yang et al., 2012, 2013a); used $m=1$ rather than $m=2$. We chose not to use $m=1$ for several reasons. First, most studies employing MSE (across imaging modalities) use $m=2$, thus allowing for more comparable parameters across studies. Second, instead of choosing parameters that minimize estimation error as recommended by Lake et al. (2002). Yang et al. (2012, 2013a); determined their parameters based on the number of regions that differed between higher and lower performing older adults. This criterion is potentially problematic because it was determined in a group of participants already showing cognitive decline, was not shown to statistically differ from other values (e.g., $m=2$ ), and being able to show greater differences between groups does not guarantee that those parameters are less free from error or bias. Lastly, on a more conceptual level, finding a pattern of two consecutive points (when using $m=1$ ) across a time series would qualify as a weak test of repeating "patterns" whereas finding a pattern of three consecutive points (when using $m=2$ ) would qualify as a stronger test of repeating patterns.

${ }^{3}$ Inspection of the noise simulations showed almost identical MSE patterns across the different networks. To test whether the noise simulations differed as a function of network, we conducted a 3 (Network) $\times 25$ (Time Scale) repeated-measures analysis of variance separately for each noise type (white, pink, and red noise). In each of these analyses (not correcting for multiple
}

BOLD signal from CSF also was extracted to evaluate the contribution of complexity within the BOLD signal that was nonneural in origin. We created a $3 \mathrm{~mm}$ sphere $(23,-39,14)$ in the posterior right ventricle to extract a time series (1) with minimal preprocessing (i.e., linear detrending only) and (2) with all of the preprocessing steps (e.g., detrending, regressing out movement, global signal, etc.).

\section{Partial Least Squares (PLS) analyses}

PLS was used in two sets of analyses to determine the extent that (1) neural complexity in each of the four RSNs differed from noise, (2) neural complexity was characterized by unique patterns in each of the four RSNs, and (3) neural complexity was associated with functional connectivity. PLS is a multivariate technique designed to identify latent factors that account for most of the variance in a data set (e.g., McIntosh et al., 1996). Because of the many time scales for each network, multivariate methods were an optimal way to capture how the pattern of network complexity in each RSN differed from noise, differed from each other, and was correlated with functional connectivity. For the first PLS analysis, the X matrix was organized in the form of (Subjects in Network $x$ Time Scale), resulting in a $180 \times 25$ matrix that allowed for the comparison between neural complexity in the RSNs and simulated noise. The Y matrix corresponded to a dummy coding of the same (Subjects in Network $\times$ Time Scale) format.

For the second analysis, separate data matrices were created for each network. The X matrix was organized in the form of (Subjects $\times$ Time Scale), resulting in a $20 \times 25$ matrix that allowed for the comparison between networks/time scales with continuous values (i.e., behavioral PLS). The Y matrix was organized in the form of (Subjects $\times$ Functional Connectivity Values), resulting in a $20 \times 2$ matrix that consisted of functional connectivity values (strength and extent) for all subjects. These connectivity values were derived from the dual regression analysis. Because we were only interested in whether functional connectivity in a given network was related to neural complexity in that network (e.g., DMN functional connectivity to DMN neural complexity), four separate PLS analyses were conducted (one for each network).

For all PLS analyses, the cross-product of the $\mathrm{X}$ and $\mathrm{Y}$ matrices was then decomposed into a set of mutually orthogonal factors using singular value decomposition, resulting in a set of orthogonal latent variables (LVs). An LV consists of three components: (1) a singular value, (2) a vector of weights representing the pattern of time scales in the LV (i.e., salience values), and (3) a vector of weights representing the degree to which each subject expresses the given LV (i.e., brain scores). Brain scores were calculated by multiplying the salience scores by the neural complexity values for each subject.

Each LV was statistically evaluated two ways. First, we assessed the significance of the network contrast represented by a given

comparisons), we found no main effects of network (all $p$ 's $>0.18$ ) and no network $\times$ scale interaction (all $p$ 's $>0.74$ ). Thus, the MSE results were averaged across the simulated networks within each subject, resulting in 25 white noise, 25 pink noise, and 25 red noise entropy values for each subject (each value corresponds to a single time scale). 
LV by determining how different the contrast was from chance and from each other. To do this, we computed 1000 permutation tests in which conditions were randomly assigned within subjects. A measure of significance was calculated by estimating the proportion of times the permuted singular value was higher than the observed singular value. Second, to assess the reliability of the corresponding distribution across subjects (i.e., saliences), we resampled subjects within conditions (1000 bootstrap samples). A bootstrap ratio (BSR) was then calculated by dividing the saliences by the standard error of the generated bootstrap distribution. The bootstrap ratio is approximately equivalent to a z-score, whereby an absolute bootstrap ratio greater than 1.96 corresponds roughly to $p<0.05$. For a given LV, positive bootstrap ratios supported the depicted contrast among conditions (e.g., $\mathrm{A}>\mathrm{B}>\mathrm{C}$ ), whereas negative bootstrap ratios supported the inverse of the contrast among conditions (e.g., $\mathrm{A}<\mathrm{B}<\mathrm{C}$ ).

\section{RESULTS}

\section{RESTING-STATE NETWORKS}

To verify that the dual regression analyses appropriately captured the four RSNs of interest, we averaged the subject-specific spatial maps (see Supplemental Figure 1). Visual inspection of these averaged maps indicated that the four networks of interest were successfully isolated.

\section{NEURAL COMPLEXITY DIFFERS FROM NOISE}

Figure 1A shows sample time courses from the BOLD signal, CSF signal, and noise simulations and Figure $\mathbf{1 B}$ shows the complexity values from the MSE analyses. The RSNs were characterized by an increase in network complexity from fine to mid time scales, followed by a slight decline in network complexity as the time scale became coarser. Even when matched on time series variance and length, the complexity of simulated noise showed very different patterns from the RSNs. White noise was characterized by a very large degree of complexity at fine time scales, which decreased exponentially with coarser time scales. Pink noise was characterized by a relatively flat level of complexity across most of the time scales with the exception of a slight increase in complexity at the first time scale. Red noise was characterized by very low level of complexity at fine time scales and a steady increase as the time scales became coarser. The CSF signal that underwent all processing steps (labeled CSF 2) showed complexity values almost identical to the white noise simulations. The CSF signal that was only detrended (labeled as CSF 1) also showed a similar pattern as white noise, but the complexity values were slightly lower at fine time scales and higher at coarse time scales, resulting in a flatter slope. Thus, the patterns of network complexity qualitatively differed from those expected due to white, pink, and red noise. In addition, network complexity differed from other physiological signals of non-neural origin (i.e., in CSF) regardless of the type of preprocessing that was conducted.

PLS analyses were conducted to quantify the differences between network complexity in the RSNs, complexity in the simulated noise, and complexity in CSF. This analysis revealed two significant LVs (both $p$ 's $<0.001$; Figure 2). The first LV (Figure 2A) explained $83.86 \%$ of the covariance and separated the RSNs from all other signals (i.e., noise and CSF) except

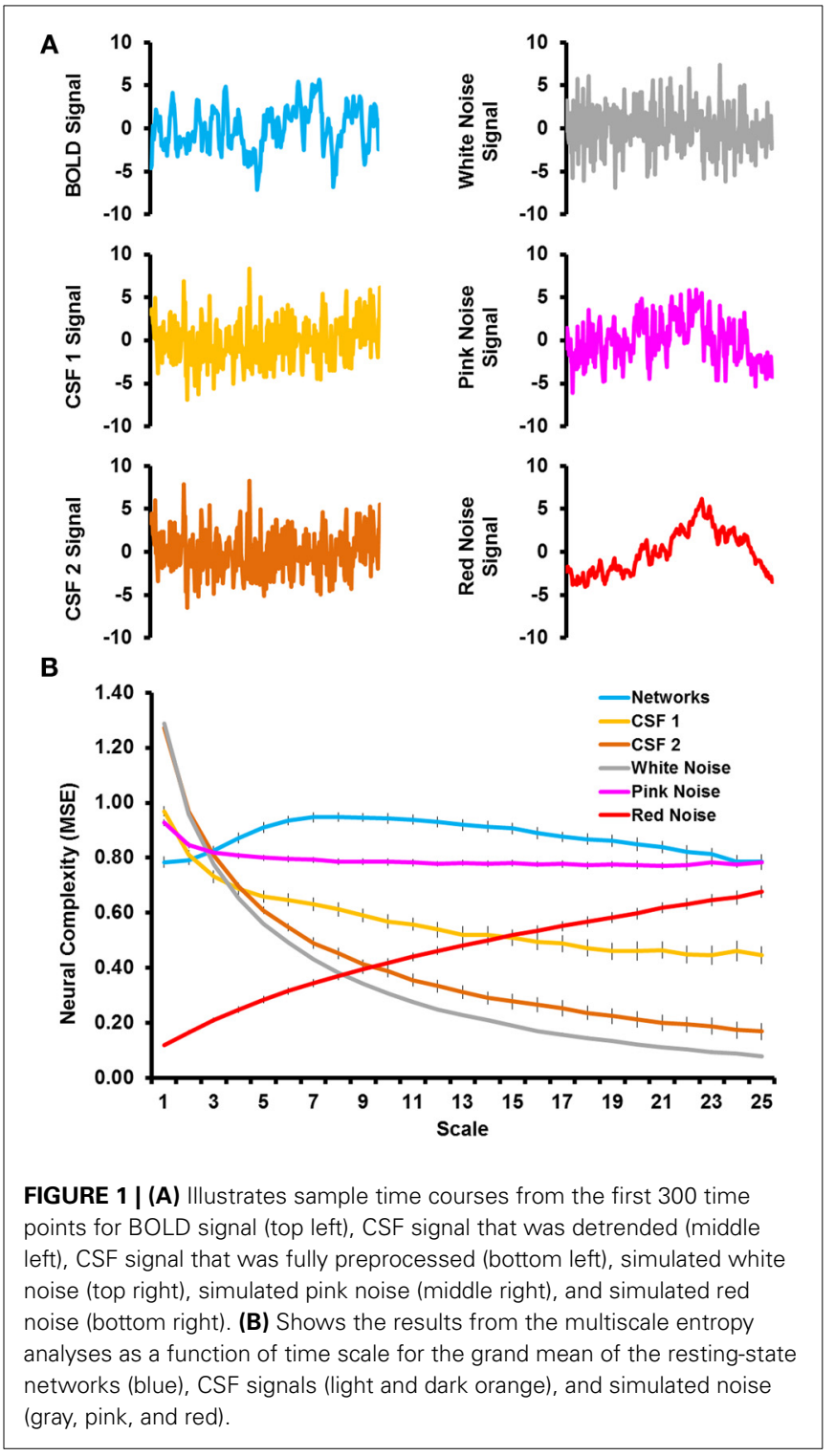

for pink noise. Although pink noise represented a pattern more similar to the RSNs, the brain scores revealed that complexity in pink noise also significantly differed from each of the RSNs. The bootstrap ratios indicated that the RSNs differed from noise at each time scale, with the third scale showing the smallest (but still reliable) difference $(B S R=12.05)$. The second LV (Figure 2B) explained $15.71 \%$ of the covariance and separated red noise from the other signals. The bootstrap rations indicated that this difference occurred at each time scale except time scale 16 $(\mathrm{BSR}=1.88)$.

\section{NETWORK COMPLEXITY DIFFERS BETWEEN RSNs}

The previous PLS analysis also indicated that each of the four RSNs differed from each other. As shown in Figure 3, network complexity was smallest in the DMN at fine time scales, but network complexity was greatest in the DMN at mid and coarse time scales relative to the other networks. The CON showed the opposite pattern; network complexity was largest in this network 


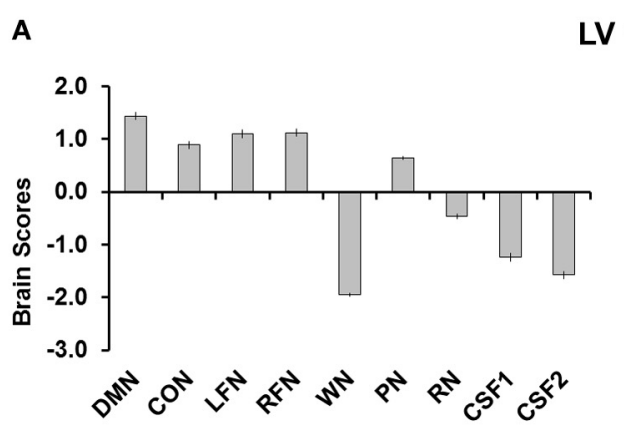

\section{1}

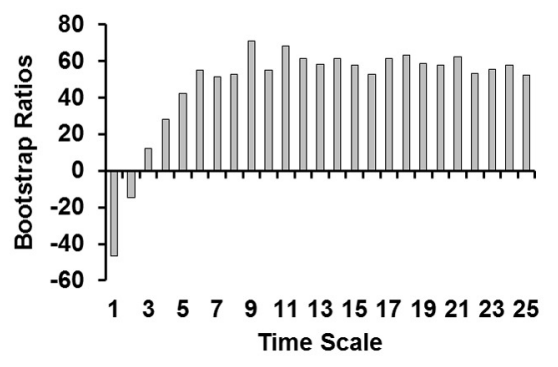

B

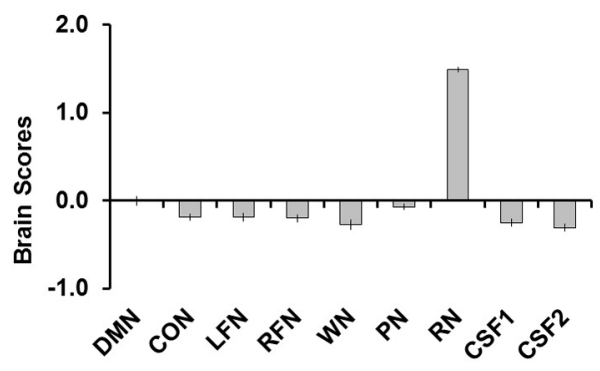

LV 2

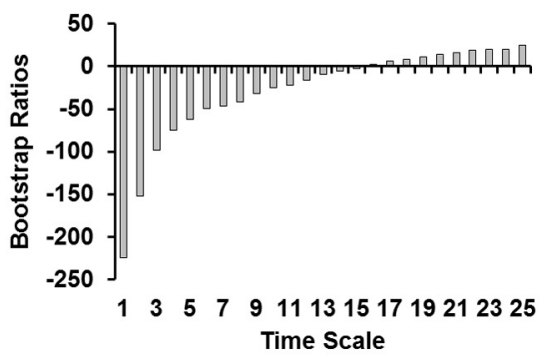

FIGURE 2 | (A) Shows the first LV and (B) shows the second LV from the PLS analysis. Brain scores are plotted on the left with error bars representing confidence intervals and the bootstrap ratios are plotted on the right to evaluate the stability of the relationships for each of the time scales. LV 1, first latent variable; LV 2, second latent variable; DMN, default mode network; CON, cingulo-opercular network; LFN, left frontoparietal network; RFN, right frontoparietal network; WN, white noise; PN, pink noise; RN, red noise; CSF1, cerebro-spinal fluid with signal detrended; CSF2, cerebro-spinal fluid signal after all preprocessing steps were completed. at fine time scales, but smallest at mid and coarse time scales. The LFN and RFN did not differ from each other, and fell in between the DMN and CON. The present results suggest that RSNs show different temporal network dynamics. One possibility, for instance, is that the DMN is characterized by less local processing and more distributed processing relative to the other networks. ${ }^{4}$

\section{NETWORK COMPLEXITY IS ASSOCIATED WITH FUNCTIONAL CONNECTIVITY \\ Default mode network}

One LV was significant $(p=0.006)$, indicating that network complexity was associated with functional connectivity in the DMN (Figure 4). The Pearson-correlation values were significant for both functional-connectivity strength $[r=0.63,95 \% \mathrm{CI}$ $(0.49,0.82)]$ and functional-connectivity extent $[r=0.62,95 \%$

\footnotetext{
${ }^{4}$ One might argue that differences in MSE across networks could be driven by differences in variance between the networks. Indeed, when calculating the variance using standard deviation (BOLD SD) in a repeated-measures ANOVA, we found a significant effect of network, $\left[F_{(3,57)}=61.94\right.$, MSE $=$ $0.138, p<0.001]$, such that BOLD SD in DMN $>$ LFN $>$ CON $=$ RFN. Interestingly, while the general patterns were similar to the MSE results (the range of MSE across time scale was largest for DMN and smallest for CON), LFN and RFN did not significantly differ in MSE, but did differ in BOLD SD, $\left[t_{(19)}=4.80, p<0.001\right]$. This deviation between MSE and BOLD SD in conjunction with the findings that the simulated noise data did not differ across networks (despite having differences in variance), suggests that variance is not driving the differences in MSE between networks.
}

CI $(0.35,0.83)]$. The salience values from the LV (Figure 4C) indicated that these correlation patterns were characterized by a negative association between network complexity and functionalconnectivity strength and extent at fine time scales, but a positive association at coarser time scales. The bootstrap ratios indicated that these associations were reliable at time scales 1,2 , and 8-25.

\section{Cingulo-opercular network}

The first LV was marginally significant $(p=0.07)$, but the Pearson-correlation values were significant for both functionalconnectivity strength $[r=0.49,95 \%$ CI $(0.34,0.74)]$ and functional-connectivity extent $[r=0.55,95 \%$ CI $(0.38,0.80)]$. The salience values from the LV indicated that these correlation patterns were characterized by a negative association between network complexity and functional connectivity-strength and extent at fine time scales, but a positive association at coarser time scales. The bootstrap ratios indicated that these associations were reliable at time scales $1-6$.

The second LV was significant $(p=0.03)$. The Pearsoncorrelation value was significant for functional-connectivity strength $[r=0.31,95 \% \mathrm{CI}(0.04,0.66)]$, but not for functionalconnectivity extent $(r=-0.15,95 \%$ CI $(-0.60,0.32)]$. The salience values from the LV indicated that these correlation patterns were characterized by a negative association between network complexity and functional-connectivity strength at fine time scales, but a positive association at coarser time scales. The bootstrap ratios indicated that these associations were reliable at time scales $1,6-9$, and 15 . 


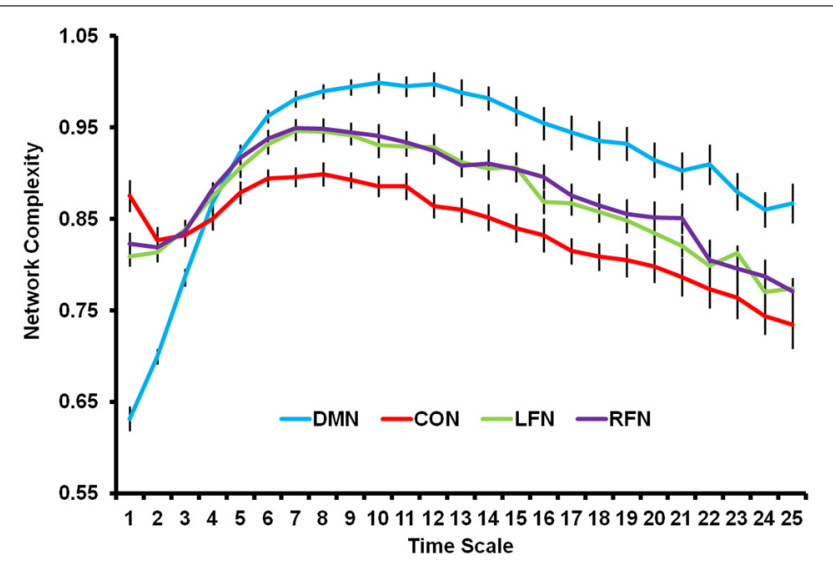

FIGURE 3 | Network complexity is plotted across time scales for the DMN, CON, LFN, and RFN. All networks showed a skewed inverted-U pattern of neural complexity across the time scales. Neural complexity at fine time scales was greatest for the CON, followed by the LFN and RFN, and was smallest for the DMN. However, neural complexity at mid and coarse time scales was greatest for the DMN, followed by the LFN and RFN, and was smallest for the CON. DMN, default mode network; CON, cingulo-opercular network; LFN, left frontoparietal network; RFN, right frontoparietal network. Error bars represent standard error of the mean.

\section{Left frontoparietal network}

Only one LV was marginally significant $(p=0.08)$ and the Pearson-correlation value was significant for functionalconnectivity strength $[r=0.61,95 \%$ CI $[0.50,0.82)]$, but not for functional-connectivity extent $(r=0.18,95 \%$ CI $[-0.16,0.54)]$. The salience values from the LV indicated that these correlation patterns were characterized by a negative association between network complexity and functional-connectivity strength at fine time scales, but a positive association at coarser time scales. The bootstrap ratios indicated that these associations were reliable at time scales $1,9,12-21$, and 25 .

\section{Right frontoparietal network}

Only one LV was significant ( $p=0.05$ ). The Pearson-correlation value was not significant for functional-connectivity strength $(r=-0.16,95 \%$ CI $[-0.44,0.51)]$, but was significant for functional-connectivity extent $(r=0.34,95 \%$ CI $[0.20,0.54)]$. The salience values from the LV indicated that these correlation patterns were characterized by a negative association between network complexity and functional-connectivity extent at fine time scales, but a positive association at coarser time scales. The bootstrap ratios indicated that these associations were reliable at time scales $4-7$.

\section{Correcting for multiple-comparisons}

To test whether network complexity was associated with functional connectivity, we conducted four separate PLS analyses. When correcting for multiple comparisons using Bonferroni correction, significance would be restricted to $p$-values $<0.013$ $(0.05 / 4)$. This stricter threshold rendered only the correlation with network complexity and the DMN significant. Nevertheless, the pattern was very similar across all networks, supporting for the robustness of the findings.

\section{DISCUSSION}

The present study aimed to better understand the complexity of brain signals in a young, healthy population using fMRI. By using a dataset with a fast sampling rate and long scanning sessions, we were able to show that neural complexity within the BOLD signal is characterized by a skewed inverted-U pattern across time scales. Specifically, neural complexity was characterized by less complexity at fine scales (i.e., more regular patterns of brain activity), a sharp increase at mid scales, followed by a slow decline in complexity as the time scale became coarser. This study is the first to show this pattern in the BOLD signal, but is often found in EEG, MEG and model simulations (e.g., Nakagawa et al., 2013). While previous fMRI studies were limited in their scope, making it difficult situate MSE in the BOLD signal with MSE in electrical activity, the present study revealed some similarities when a high sampling rate and longer epochs were employed. Furthermore, the present study expanded upon previous research by showing that neural complexity in the BOLD signal differed from noise, differed from non-neural signals, differed between RSNs, and was correlated with functional connectivity. We elaborate on these key findings below.

\section{DIFFERENTIATING NEURAL COMPLEXITY FROM NOISE}

Neural complexity was estimated by measures of sample entropy, or the degree of randomness within a signal. While greater complexity has generally been associated with better cognition (e.g., McIntosh et al., 2008; Sokunbi et al., 2011; Yang et al., 2012), health (Yang and Tsai, 2013), and maturing brain systems (e.g., McIntosh et al., 2008; Vakorin et al., 2011), signals of pure noise also are highly random. Thus, signals containing meaningful information must be differentiated from signals varying randomly. Few studies have compared random patterns of fluctuations with complex patterns within the BOLD signal. However, one fMRI study conducted by Smith et al. (2013) compared complexity values of pink and white noise with the BOLD signal. They found that pink noise was characterized by similar levels of complexity across all time scales (i.e., resembling a flat line), and this pattern largely differed from the BOLD signal, which showed an exponential decrease in complexity values from fine to coarse time scales. The patterns of pink noise in the present study resembled the Smith et al. (2013) study, showing similar levels of complexity across time scales, which differed from BOLD signal.

In contrast to pink noise, Smith et al. (2013) found that complexity values in white noise did not differ from those in the BOLD signal at fine time scales, but rather began to differentiate as the time scales became coarser. Also striking in their study was the similar pattern of complexity between white noise and the BOLD signal; complexity values started high and exponentially decreased at similar rates. While results from the present study also showed patterns of complexity in white noise that exponentially decreased with increasing time scales, complexity in white noise differed from neural complexity in the BOLD signal at each time scale. One clear difference between the present study and the study conducted by Smith et al. (2013) is the greater temporal resolution and longer sessions in the present study, allowing for more precise estimations of complexity. Another key difference was that Smith et al. (2013) averaged complexity values across 


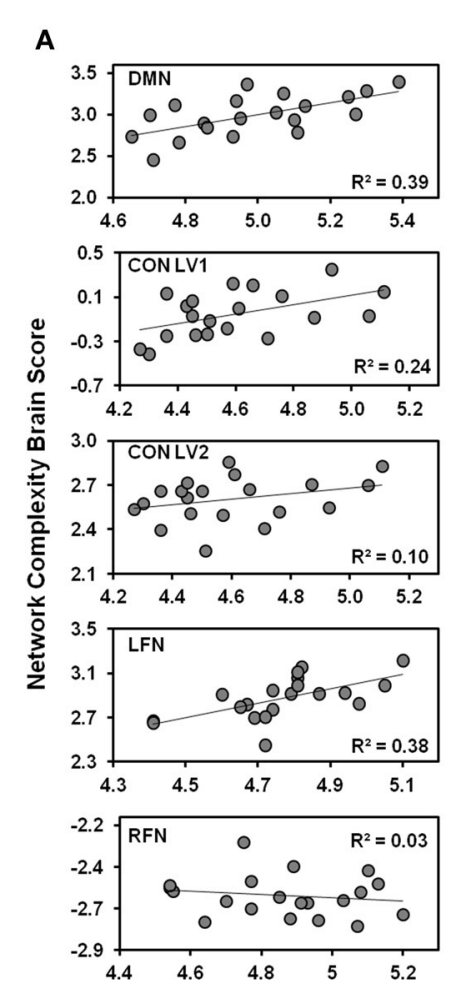

Functional Connectivity Strength $(Z)$
B
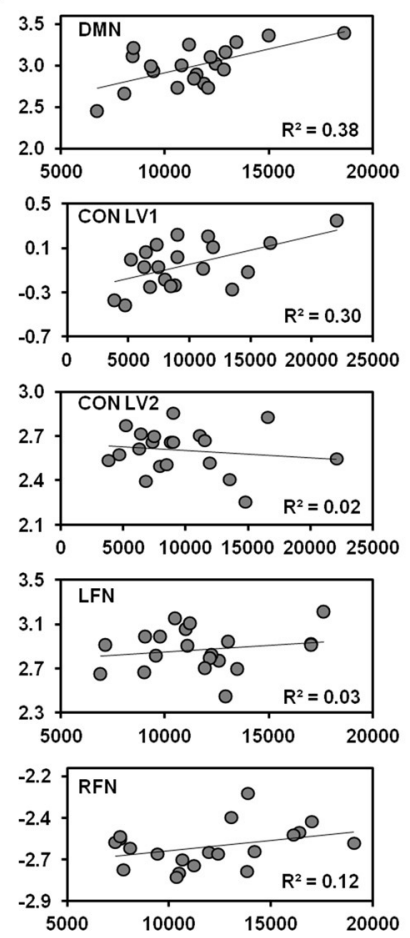

Functional Connectivity Extent (Voxels)

C

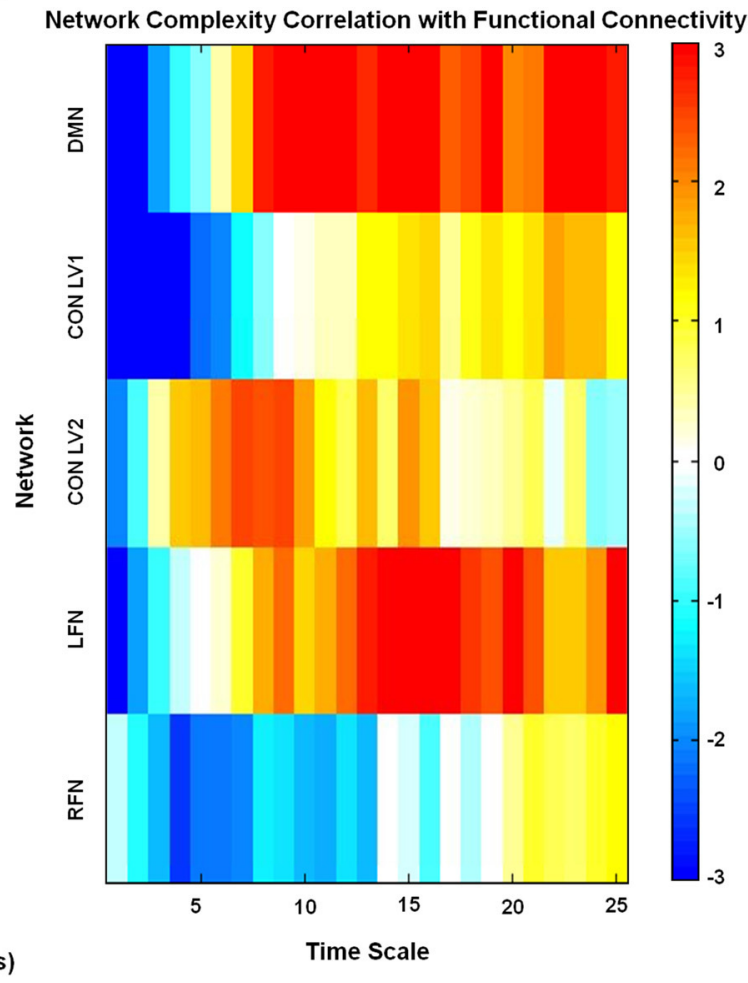

FIGURE 4 | The relationship is plotted between network complexity and functional connectivity. (A) Shows the correlations between functional connectivity strength ( $x$-axis) and the network complexity brain scores from the PLS analysis (y-axis). (B) Shows the correlations between functional connectivity extent ( $x$-axis) and the network complexity brain scores from the PLS analysis (y-axis). The brain scores represent the pattern of network complexity across time scales as shown in (C). Cool colors represent a negative association and hot colors represent a positive association between functional connectivity and network complexity at a given time scale. Network complexity within each network exhibits a similar relationship with functional connectivity; negative relationships were found at fine time scales and positive relationships were found at coarser time scales. DMN, default mode network; CON, cingulo-opercular network; LFN, left frontoparietal network; RFN, right frontoparietal network; LV1, latent variable 1; LV2, latent variable 2. all gray matter voxels, whereas the present study only assessed gray matter voxels within networks associated with higher-order cognition. Averaging across different networks may have led to a mixing of different patterns of neural complexity, and thus a different pattern from the current study. Along these lines, we did find evidence that different RSNs were characterized by significantly different levels of complexity at multiple time scales. The complexity patterns of white noise also resembled the complexity patterns within CSF, supporting the idea that complexity within RSNs are neural in origin. Lastly, this study is the first to compare simulations of red noise, which contains spectral properties similar to the BOLD signal (but with a different exponent than pink noise). Unlike white and pink noise, red noise differed the most from complexity in the RSNs at fine time scales and became more similar at coarse time scales. Together, these studies suggest that some time scales may be dominated by random noise rather than meaningful signal. Because acquisition parameters often differ across studies, assessing noise is important to make meaningful interpretations of neural complexity.

\section{CHARACTERIZING NETWORK COMPLEXITY ACROSS RSNs}

Neural complexity should differ between RSNs to the extent that the complexity represents the temporal dynamics within that system, possibly relating to the interconnectivity among local neural populations and long-range interactions across distributed neural populations (Mizuno et al., 2010; Vakorin et al., 2011; McIntosh et al., 2013). The most striking finding across networks was that the DMN showed the smallest degrees of network complexity at fine scales, but the largest degrees of network complexity at mid and coarse scales relative to the other networks. Because fluctuations of brain activity while at rest largely represent the history of co-activations between regions (e.g., Wig et al., 2011), this observation would suggest that the DMN consists of greater degrees of information processing across distributed connections (e.g., medial frontal and medial parietal regions) relative to other networks. In contrast to the DMN, the other networks did not have as large of a range of complexity across time scales, suggesting relatively similar levels of information processing among both local and distributed connections. Interestingly, the CON showed the greatest degree of network complexity at fine scales, but the smallest degree of network complexity at coarse scales.

The correlations between network complexity and functional connectivity can help understand how to interpret the differences in complexity between RSNs at fine and coarse time scales. The network complexity across all of the RSNs showed a consistent 
pattern; network complexity was negatively correlated with both the strength and extent of functional connectivity at fine time scales, but was positively correlated at coarse time scales. This pattern is most consistent with the proposal that neural complexity is related to the regulation of neural synchrony (section Regulation of Neural Synchrony) and with the ideas that that information processing is maximized when neurons desynchronize at fine time scales, but synchronize at coarse time scales, consistent with neural models showing that time scale is critical to understand the neural dynamics involved in information transfer between neurons (Baptista and Kurths, 2008). Thus, one interpretation of the present results is that the DMN shows both the greatest degrees of desynchrony at fine scales and synchrony at coarse time scales relative to the other networks.

This interpretation is consistent with the idea that regions within the DMN serve as hub centers that act as critical gateways for information processing that integrate diverse sources of information within local and across distributed networks (e.g., Mesulam, 1998; Bassett and Bullmore, 2006; Buckner et al., 2009). For example, the DMN also is associated with many domains of cognition, and therefore information is constantly being exchanged within and across this network. Indeed, the default mode network has been related to episodic memory, imagining the future, self-reflection, mentalizing, divergent thinking, working memory, reading comprehension, and constructing moral judgments (Hampson et al., 2006; Buckner et al., 2008; Li et al., 2009; Spreng et al., 2009; van den Heuvel et al., 2009).

The fact that the other RSNs showed greater network complexity at fine scales, but less network complexity at coarse scales may be related to network dynamics at rest compared with online processing. For instance, activity in the DMN is often elevated at rest, but suppressed during tasks - especially those requiring cognitive control (e.g., Shulman et al., 1997; Mazoyer et al., 2001; Raichle et al., 2001). This pattern is consistent with the current study in that the DMN shows the greatest range in neural complexity at rest. Because the CON, LFN, and RFN have been implicated in online processing (i.e., are task positive networks), it is possible that the range of network complexity might change during a task. Thus, depending on the exact task and degree of cognitive control exerted, these networks might show the greatest range in neural complexity (i.e., smallest level of complexity at fine scales, but greatest level of complexity at coarse scales). No study to our knowledge has assessed neural complexity using MSE at rest and during a task-a possible avenue for future work.

\section{ALTERNATIVE INTERPRETATIONS OF NEURAL COMPLEXITY}

While the results from the present findings largely support the idea that neural complexity is involved in the regulation of neural synchrony (or asynchrony), other interpretations cannot be entirely ruled out. These other interpretations, however, require additional explanations or evidence that is outside the scope of the present study. Specifically, an alternative idea is that neural complexity might represent the range or capacity of the brain to explore alternative brain states (for review, see Garrett et al., 2013; see section A Dynamic Range of Microstates). This idea proposes that randomness in brain activity arises from the constant fluctuation or transition of different brain states. To the extent that these transitions create complex patterns and are associated with information processing, then only low frequency signals at coarse time scales represent these types of transitioning neural dynamics. This theory does not explain the negative correlations between network complexity and functional connectivity at fine time scales. Another idea is that the randomness of fluctuating brain activity represents a moderate level of noise in a system that enhances the probability of neuronal firing (for review, see Garrett et al., 2013; see section Facilitation of Neuronal Firing). The current findings are consistent with this idea to the extent that what constitutes a moderate degree of noise differs between fine and coarse time scales. Less noise (and thus more regular brain activity) would play an optimal role to facilitate neuronal firing at fine scales, but more noise (and thus more random brain activity) would facilitate neuronal firing at coarse scales. However, it is not clear how these different levels of noise would interact with time scales in this manner.

While more work still needs to be done to fully understand how neural complexity fits in with the larger context of neural dynamics, a few key questions remain outstanding. First, do different degrees of neural complexity establish an environment that facilitates functional connectivity as proposed by Ghanbari et al. (2013)? This idea suggests that neural complexity does not represent information processing, per se, but rather influences the conditions under which optimal information processing can occur. Specifically, Ghanbari et al. (2013) proposed that the synchrony between any two nodes is more likely when signals are more predictable, thus having less complexity. While our findings only support this idea at fine time scales, the possibility remains that neural complexity does not directly correspond to the degree of information processing.

On the other hand, it has been suggested that neural complexity does relate more directly to the richness of information (e.g., Tononi et al., 1994, 1998; Nakagawa et al., 2013) or the amount of information integration (e.g., Vakorin et al., 2011; McIntosh et al., 2013) within a network. Evidence supporting this idea comes from estimates of neural complexity during different task conditions. For instance, neural complexity has been found to be greater during conditions when eyes are open compared with conditions when eyes are closed (Hogan et al., 2012), when learned information is highly familiar compared with less familiar (Heisz et al., 2012), when retrieving episodic information compared with semantic information (Heisz et al., 2013), and when faces are processed inverted compared with upright (Mišić et al., 2010). This idea would suggest that neural complexity is directly influenced by online processing and dynamically changes depending on concurrent cognitive processes.

\section{IMPLICATIONS FOR AGING AND CLINICAL DISORDERS}

One of the goals of the present study was to assess neural complexity in a population that was young and healthy to better understand the pattern of findings in populations of different ages and clinical disorders. A common notion is that greater neural complexity is associated with healthier and more functional states. Thus, it is inferred that a decrease in neural complexity is associated with clinical disorders (cf. Yang and Tsai, 2013). 
The present results speak against such broad conclusions. At fine time scales-which would include most fMRI studieslow levels of neural complexity might be optimal for maximum information processing. Specifically, we found that decreases in neural complexity at fine time scales were associated with greater functional connectivity in a healthy sample across four key networks associated with higher-order cognitive processing. Thus, increases in neural complexity at fine time scales might actually be associated with a deficit in information processing. This pattern can inform recent studies investigating the effects of neural complexity and functional connectivity in populations at risk for developing Alzheimer's disease. For instance, a recent fMRI study showed that older adults who carried the APOE E4 allele-a risk for developing Alzheimer's disease-had less neural complexity in precuneus and posterior cingulate than non-carriers and also had increased functional connectivity in these regions with frontal regions compared with non-carriers (Yang et al., 2013a). Because young, healthy adults also show this relationship, it might be inferred that the decreases in neural complexity is associated with more information processing, potentially as a compensatory mechanism to counteract structural or other neurobiological declines (cf. Park and Reuter-Lorenz, 2009) stemming from having the APOE E4 allele (e.g., Poirier et al., 1993; Bookheimer et al., 2000; Small et al., 2000; Mahley et al., 2006). This interpretation differs from the general notion that decreases in neural complexity is associated with dysfunction in a system.

Other studies using EEG and patients with probable Alzheimer's disease also have shown decreases in neural complexity at fine scales. Furthermore, increases in neural complexity at coarse scales relative to healthy controls are evident (e.g., Escudero et al., 2006; Mizuno et al., 2010; Yang et al., 2013b). In light of the present findings, both the decreased levels of neural complexity at fine scales and the increased levels of neural complexity at coarse scales would be interpreted as corresponding to increased levels of functional connectivity. However, one assumption to this line of reasoning is that greater connectivity is always beneficial. While often times it might be beneficial-especially if healthy, young adults show a similar pattern-increases levels of connectivity might be detrimental (i.e., hyper connectivity; e.g., Bai et al., 2011; Mayer et al., 2011; Fornito et al., 2012). By relating both neural complexity and functional connectivity measures to behavioral performance, one can better assess whether these differences are associated with compensation or dysfunction.

A related and more neutral interpretation of neural complexity differences (without assuming increases or decreases are necessarily associated with a dysfunctional system) is that greater neural complexity at fine scales is associated with a bias away from interconnectivity within local connections, whereas greater neural complexity at coarse scales is associated with a bias toward distributed connectivity between long-range connections. Similar arguments have been made in the context healthy aging. For example, McIntosh et al. (2013) investigated age differences in MSE and functional connectivity using both EEG and MEG. At fine scales, they found age-related increases in both complexity and within-hemisphere (i.e., local) connectivity. At coarse scales, the reverse occurred; they found age-related decreases in both complexity and across-hemisphere (distributed) connectivity.

\section{LIMITATIONS}

There are some limitations in directly comparing these findings to studies using MSE in different imaging modalities. While the pattern of complexity in the current study is similar to that found in EEG and MEG studies, the origin of the brain signals still differs between modalities (i.e., electrical vs. BOLD activity) and thus the neural dynamics underlying complexity across time scales might also differ. For example, the study comparing young and old adults using EEG and MEG conducted by McIntosh et al. (2013) found both age-related increases in neural complexity and functional connectivity at fine time scales, which suggests a positive relationship between these two measures. If a positive relationship does exist between these measures in EEG and MEG, then neural complexity may be tapping into different underlying mechanisms than neural complexity in fMRI. While this might indeed be the case, that study did not directly correlate the two measures together, thus it could be the case that while aging does affect the overall level of neural complexity and connectivity, individual differences within each age group could still show a negative relationship.

Another limitation is that very few studies have analyzed MSE in fMRI, making the underlying neural mechanisms of MSE unclear. As outlined in the Introduction (section Neural Complexity: Theories and Evidence), differences in network complexity could be related to the range of microstates, facilitative noise in a system, or neural synchrony (local and distributed information processing). While we favor an interpretation of neural synchrony (for discussion, see section Alternative Interpretations of Neural Complexity), it is still possible that the type of "local processing" captured at fine time scales in EEG/MEG might be qualitatively different from the type of "local processing" captured at fine time scales in the current study. Nevertheless, the current study was able to capture a large range of time scales comparable to most EEG/MEG studies, suggesting that the relative differences in local vs. distributed processing remain similar. This idea is supported by the pattern of MSE that shows a low-value of network complexity at fine time scales, followed by a rapid increase in network complexity-a similar pattern to that found in EEG and MEG studies, suggesting that we might be tapping into local processing. Future research is needed to verify that the underlying network dynamics captured by MSE is similar across different imaging modalities.

\section{CONCLUSION}

Neural complexity provides novel insights into the neural dynamics underlying information processing, but the neural complexity across different RSNs in healthy young adults has largely been ignored. The present study provided evidence that the complexity within BOLD signals differs from random fluctuations associated with noise, differs between RSNs, and that complexity is associated with both the strength and extent of functional connectivity across RSNs. These findings complement other analysis techniques aimed to measure information processing and can help better understand both healthy brain systems and abnormal 
brain systems including aged and clinical populations. Contrary to growing beliefs, less neural complexity is not always indicative of declines in information processing. Only by further exploring the brain's temporal dynamics across a variety of contexts will we come to fully understand neural complexity and its relationship with cognition and pathological states.

\section{ACKNOWLEDGMENTS}

Data from the Human Connectome Project, WU-Minn Consortium (Principal Investigators: David Van Essen and Kamil Ugurbil; 1U54MH091657) were funded by the 16 NIH Institutes and Centers that support the NIH Blueprint for Neuroscience Research; and by the McDonnell Center for Systems Neuroscience at Washington University. We thank Gérard N. Bischof and Sara Haber for comments on previous versions of the manuscript.

\section{SUPPLEMENTARY MATERIAL}

The Supplementary Material for this article can be found online at: http://www.frontiersin.org/journal/10.3389/fnhum. 2014.00409/abstract

Supplemental Figure 1 | The four resting-state networks of interest were successfully isolated using a priori templates in dual regression analyses. DMN, default mode network; CON, cingulo-opercular network; LFN, left frontoparietal network; RFN, right frontoparietal network.

\section{REFERENCES}

Andersson, J. L. R., Skare, S., and Ashburner, J. (2003). How to correct susceptibility distortions in spin-echo echo-planar images: application to diffusion tensor imaging. Neuroimage 20, 870-888. doi: 10.1016/S1053-8119(03)00336-7

Bai, F., Watson, D. R., Shi, Y., Wang, Y., Yue, C., YuhuanTeng, W. D., et al. (2011). Specifically progressive deficits of brain functional marker in amnestic type mild cognitive impairment. PLoS ONE 6:e24271. doi: 10.1371/journal.pone.0024271

Baptista, M. S., and Kurths, J. (2008). Transmission of information in active networks. Phys. Rev. E 77:026205. doi: 10.1103/PhysRevE.77.026205

Bassett, D. S., and Bullmore, E. (2006). Small-world brain networks. Neuroscientist 12, 512-523. doi: 10.1177/1073858406293182

Beckmann, C. F., Mackay, C. E., Filippini, N., and Smith, S. M. (2009). Group comparison of rest- ing-state fMRI data using multi-subject ICA and dual regression. Neuroimage 47(Suppl. 1), S148. doi: 10.1016/S1053-8119(09) 71511-3

Beckmann, C. F., and Smith, S. M. (2004). Probabilistic independent component analysis for functional magnetic resonance imaging. IEEE Trans. Med. Imaging 23, 137-152. doi: 10.1109/TMI.2003.822821

Beharelle, A. R., Kovaèević, N., McIntosh, A. R., and Levine, B. (2012). Brain signal variability relates to stability of behavior after recovery from diffuse brain injury. Neuroimage 60, 1528-1537. doi: 10.1016/j.neuroimage.2012.01.037

Biswal, B. B., Mennes, M., Zuo, X.-N., Gohel, S., Kelly, C., Smith, S. M., et al. (2010). Toward discovery science of human brain function. Proc. Natl. Acad. Sci. U.S.A. 107, 4734-4739. doi: 10.1073/pnas.0911855107

Bookheimer, S. Y., Strojwas, M. H., Cohen, M. S., Saunders, A. M., PericakVance, M. A., Mazziotta, J. C., et al. (2000). Patterns of brain activation in people at risk for Alzheimer's disease. N. Engl. J. Med. 343, 450-456. doi: 10.1056/NEJM200008173430701

Bosl, W., Tierney, A., Tager-Flusberg, H., and Nelson, C. (2011). EEG complexity as a biomarker for autism spectrum disorder risk. BMC Med. 9:18. doi: 10.1186/1741-7015-9-18

Buckner, R. L., Andrews-Hanna, J. R., and Schacter, D. L. (2008). The brain's default network: anatomy, function, and relevance to disease. Ann. N. Y. Acad. Sci. 1124, 1-38. doi: 10.1196/annals.1440.011

Buckner, R. L., Sepulcre, J., Talukdar, T., Krienen, F. M., Liu, H., Hedden, T., et al. (2009). Cortical hubs revealed by intrinsic functional connectivity: mapping, assessment of stability, and relation to Alzheimer's disease. J. Neurosci. 29, 1860-1873. doi: 10.1523/JNEUROSCI.5062-08.2009
Bullmore, E., Fadili, J., Maxim, V., Sendur, L., Whitcher, B., Suckling, J., et al. (2004). Wavelets and functional magnetic resonance imaging of the human brain. Neuroimage 23, S234-S249. doi: 10.1016/j.neuroimage.2004. 07.012

Calhoun, V., and Adali, T. (2012). Multisubject independent component analysis of fMRI: a decade of intrinsic networks, default mode, and neurodiagnostic discovery. IEEE Rev. Biomed. Eng. 5, 60-73. doi: 10.1109/RBME.2012.22 11076

Catarino, A., Churches, O., Baron-Cohen, S., Andrade, A., and Ring, H. (2011). Atypical EEG complexity in autism spectrum conditions: a multiscale entropy analysis. Clin. Neurophysiol. 122, 2375-2383. doi: 10.1016/j.clinph.2011. 05.004

Costa, M., Goldberger, A. L., and Peng, C. K. (2005). Multiscale entropy analysis of biological signals. Phys. Rev. E Stat. Nonlin. Soft Matter Phys. 71:021906. doi: 10.1103/PhysRevE.71.021906

Deco, G., Jirsa, V. K., and McIntosh, A. R. (2011). Emerging concepts for the dynamical organization of resting-state activity in the brain. Nat. Rev. Neurosci. 12, 43-56. doi: 10.1038/nrn2961

Dosenbach, N. U. F., Fair, D. A., Cohen, A. L., Schlaggar, B. L., and Petersen, S. E. (2008). A dual-networks architecture of top-down control. Trends Cogn. Sci. 12, 99-105. doi: 10.1016/j.tics.2008.01.001

Escudero, J., Abásolo, D., Hornero, R., Espino, P., and López, M. (2006). Analysis of electroencephalograms in Alzheimer's disease patients with multiscale entropy. Physiol. Meas. 27, 1091-1106. doi: 10.1088/0967-3334/27/11/004

Faisal, A. A., Selen, L. P. J., and Wolpert, D. M. (2008). Noise in the nervous system. Nat. Rev. Neurosci. 9, 292-303. doi: 10.1038/nrn2258

Filippini, N., MacIntosh, B. J., Hough, M. G., Goodwin, G. M., Frisoni, G. B., Smith, S. M., et al. (2009). Distinct patterns of brain activity in young carriers of the APOE-epsilon4 allele. Proc. Natl. Acad. Sci. U.S.A. 106, 7209-7214. doi: 10.1073/pnas.0811879106

Fornito, A., Zalesky, A., Pantelis, C., and Bullmore, E. T. (2012). Schizophrenia, neuroimaging and connectomics. Neuroimage 62, 2296-2314. doi: 10.1016/j.neuroimage.2011.12.090

Friston, K., Breakspear, M., and Deco, G. (2012). Perception and self-organized instability. Front. Comput. Neurosci. 6:44. doi: 10.3389/fncom.2012.00044

Garrett, D. D., Samanez-Larkin, G. R., MacDonald, S. W. S., Lindenberger, U., McIntosh, A. R., and Grady, C. L. (2013). Moment-to-moment brain signal variability: a next frontier in human brain mapping? Neurosci. Biobehav. Rev. 37, 610-624. doi: 10.1016/j.neubiorev.2013.02.015

Ghanbari, Y., Bloy, L., Christopher Edgar, J., Blaskey, L., Verma, R., and Roberts, T. P. L. (2013). Joint analysis of band-specific functional connectivity and signal complexity in autism. J. Autism Dev. Disord. doi: 10.1007/s10803-013-1915-7. [Epub ahead of print].

Ghosh, A., Rho, Y., McIntosh, A. R., Kötter, R., and Jirsa, V. K. (2008). Noise during rest enables the exploration of the brain's dynamic repertoire. PLoS Comput. Biol. 4:e1000196. doi: 10.1371/journal.pcbi.1000196

Glasser, M. F., Sotiropoulos, S. N., Wilson, J. A., Coalson, T. S., Fischl, B., Andersson, J. L., et al. (2013). The minimal preprocessing pipelines for the Human Connectome Project. Neuroimage 80, 105-124. doi: 10.1016/j.neuroimage.2013.04.127

Goldberger, A. L., Peng, C.-K., and Lipsitz, L. A. (2002). What is physiologic complexity and how does it change with aging and disease? Neurobiol. Aging 23, 23-26. doi: 10.1016/S0197-4580(01)00266-4

Gomez, C., Poza, J., Fernandez, A., Bachiller, A., Gomez, J., and Hornero, R. (2013). Entropy analysis of MEG background activity in AttentionDeficit/Hyperactivity Disorder. Conf. Proc. IEEE Eng. Med. Biol. Soc. 2013, 5057-5060. doi: 10.1109/EMBC.2013.6610685

Hampson, M., Driesen, N. R., Skudlarski, P., Gore, J. C., and Constable, R. T. (2006). Brain connectivity related to working memory performance. J. Neurosci. 26, 13338-13343. doi: 10.1523/JNEUROSCI.3408-06.2006

He, B. J. (2011). Scale-free properties of the functional magnetic resonance imaging signal during rest and task. J. Neurosci. 31, 13786-13795. doi: 10.1523/JNEUROSCI.2111-11.2011

He, B. J., Zempel, J. M., Snyder, A. Z., and Raichle, M. E. (2010). The temporal structures and functional significance of scale-free brain activity. Neuron 66, 353-369. doi: 10.1016/j.neuron.2010.04.020

Heisz, J. J., Shedden, J. M., and McIntosh, A. R. (2012). Relating brain signal variability to knowledge representation. Neuroimage 63, 1384-1392. doi: 10.1016/j.neuroimage.2012.08.018 
Heisz, J. J., Vakorin, V., Ross, B., Levine, B., and McIntosh, A. R. (2013). A Trade-off between local and distributed information processing associated with remote episodic versus semantic memory. J. Cogn. Neurosci. 26, 41-53. doi: 10.1162/jocn_a_00466

Hogan, M. J., Kilmartin, L., Keane, M., Collins, P., Staff, R. T., Kaiser, J., et al. (2012). Electrophysiological entropy in younger adults, older controls and older cognitively declined adults. Brain Res. 1445, 1-10. doi: 10.1016/j.brainres.2012.01.027

Honey, C. J., Kötter, R., Breakspear, M., and Sporns, O. (2007). Network structure of cerebral cortex shapes functional connectivity on multiple time scales. Proc. Natl. Acad. Sci. U.S.A. 104, 10240-10245. doi: 10.1073/pnas.0701519104

Honey, C. J., Sporns, O., Cammoun, L., Gigandet, X., Thiran, J. P., Meuli, R., et al. (2009). Predicting human resting-state functional connectivity. Proc. Natl. Acad. Sci. U.S.A. 106, 2035-2040. doi: 10.1073/pnas.0811168106

Jenkinson, M., Bannister, P., Brady, M., and Smith, S. (2002). Improved optimization for the robust and accurate linear registration and motion correction of brain images. Neuroimage 17, 825-841. doi: 10.1006/nimg.2002.1132

Jenkinson, M., Beckmann, C. F., Behrens, T. E., Woolrich, M. W., and Smith, S. M. (2012). FSL. Neuroimage 62, 782-790. doi: 10.1016/j.neuroimage.2011.09.015

Kiviniemi, V., Kantola, J.-H., Jauhiainen, J., Hyvärinen, A., and Tervonen, O. (2003). Independent component analysis of nondeterministic fMRI signal sources. Neuroimage 19, 253-260. doi: 10.1016/S1053-8119(03)00097-1

Laird, A. R., Fox, P. M., Eickhoff, S. B., Turner, J. A., Ray, K. L., McKay, D. R., et al. (2011). Behavioral interpretations of intrinsic connectivity networks. J. Cogn. Neurosci. 23, 4022-4037. doi: 10.1162/jocn_a_00077

Lake, D. E., Richman, J. S., Griffin, M. P., and Moorman, J. R. (2002). Sample entropy analysis of neonatal heart rate variability. Am. J. Physiol. Regul. Integr. Comp. Physiol. 283, R789-R797. doi: 10.1152/ajpregu.00069.2002

Li, Y., Liu, Y., Li, J., Qin, W., Li, K., Yu, C., et al. (2009). Brain anatomical network and intelligence. PLoS Comput. Biol. 5:e1000395. doi: 10.1371/journal.pcbi. 1000395

Mahley, R. W., Weisgraber, K. H., and Huang, Y. (2006). Apolipoprotein E4: a causative factor and therapeutic target in neuropathology, including Alzheimer's disease. Proc. Natl. Acad. Sci. U.S.A. 103, 5644-5651. doi: 10.1073/pnas.0600549103

Mayer, A. R., Mannell, M. V., Ling, J., Gasparovic, C., and Yeo, R. A. (2011). Functional connectivity in mild traumatic brain injury. Hum. Brain Mapp. 32, 1825-1835. doi: 10.1002/hbm.21151

Mazoyer, B., Zago, L., Mellet, E., Bricogne, S., Etard, O., Houdé, O., et al. (2001). Cortical networks for working memory and executive functions sustain the conscious resting state in man. Brain Res. Bull. 54, 287-298. doi: 10.1016/S03619230(00)00437-8

McDonnell, M. D., and Ward, L. M. (2011). The benefits of noise in neural systems: bridging theory and experiment. Nat. Rev. Neurosci. 12, 415-426. doi: 10.1038/nrn3061

McIntosh, A. R., Bookstein, F. L., Haxby, J. V., and Grady, C.L. (1996). Spatial pattern analysis of functional brain images using partial least squares. Neuroimage 3, 143-157. doi: 10.1006/nimg.1996.0016

McIntosh, A. R., Kovacevic, N., and Itier, R. J. (2008). Increased brain signal variability accompanies lower behavioral variability in development. PLoS Comput. Biol. 4:e1000106. doi: 10.1371/journal.pcbi.1000106

McIntosh, A. R., Kovacevic, N., Lippe, S., Garrett, D., Grady, C., and Jirsa, V. (2010). The development of a noisy brain. Arch. Ital. Biol. 148, 323-337. doi: 10.4449/ aib.v148i3.1225

McIntosh, A. R., Vakorin, V., Kovacevic, N., Wang, H., Diaconescu, A., and Protzner, A. B. (2013). Spatiotemporal dependency of age-related changes in brain signal variability. Cereb. Cortex. doi: 10.1093/cercor/bht030. [Epub ahead of print].

Méndez, M., Zuluaga, P., Hornero, R., Gómez, C., Escudero, J., Rodríguez-Palancas, A., et al. (2012). Complexity analysis of spontaneous brain activity: effects of depression and antidepressant treatment. J. Psychopharmacol. 26, 636-643. doi: 10.1177/0269881111408966

Menon, V., and Uddin, L. Q. (2010). Saliency, switching, attention and control: a network model of insula function. Brain Struct. Funct. 214, 655-667. doi: 10.1007/s00429-010-0262-0

Mesulam, M. M. (1998). From sensation to cognition. Brain 121, 1013-1052. doi: 10.1093/brain/121.6.1013

Milstein, J., Mormann, F., Fried, I., and Koch, C. (2008). Neuronal shot noise and Brownian 1/f2 behavior in the local field potential. PLOS ONE 4:e4338. doi: 10.1371/journal.pone.0004338
Mišić, B., Mills, T., Taylor, M. J., and McIntosh, A. R. (2010). Brain noise is task dependent and region specific. J. Neurophys. 104, 2667-2676. doi: 10.1152/jn.00648.2010

Mišić, B., Vakorin, V. A., Paus, T., and McIntosh, A. R. (2011). Functional embedding predicts the variability of neural activity. Front. Syst. Neurosci. 5:90. doi: $10.3389 /$ fnsys. 2011.00090

Mizuno, T., Takahashi, T., Cho, R. Y., Kikuchi, M., Murata, T., Takahashi, K., et al. (2010). Assessment of EEG dynamical complexity in Alzheimer's disease using multiscale entropy. Clin. Neurophysiol. 121, 1438-1446. doi: 10.1016/j.clinph.2010.03.025

Murphy, K., Birn, R. M., Handwerker, D. A., Jones, T. B., and Bandettini, P. A. (2009). The impact of global signal regression on resting state correlations: are anti-correlated networks introduced? Neuroimage 44, 893-905. doi: 10.1016/j.neuroimage.2008.09.036

Nakagawa, T. T., Jirsa, V. K., Spiegler, A., McIntosh, A. R., and Deco, G. (2013). Bottom up modeling of the connectome: linking structure and function in the resting brain and their changes in aging. Neuroimage 80, 318-329. doi: 10.1016/j.neuroimage.2013.04.055

Park, D. C., and Reuter-Lorenz, P. (2009). The adaptive brain: aging and neurocognitive scaffolding. Annu. Rev. Psychol. 60, 173-196. doi: 10.1146/annurev.psych.59.103006.093656

Pincus, S. M. (1991). Approximate entropy as a measure of system complexity. Proc. Natl. Acad. Sci. U.S.A. 88, 2297-2301. doi: 10.1073/pnas.88.6.2297

Pincus, S. M., and Goldberger, A. L. (1994). Physiological time-series analysis: what does regularity quantify? Am. J. Physiol. 266, H1643-H1656.

Poirier, J., Bertrand, P., Kogan, S., Gauthier, S., Davignon, J., and Bouthillier, D. (1993). Apolipoprotein E polymorphism and Alzheimer's disease. Lancet 342, 697-699. doi: 10.1016/0140-6736(93)91705-Q

Posner, J., Park, C., and Wang, Z. (2014). Connecting the dots: a review of resting connectivity MRI studies in attention-deficit/hyperactivity disorder. Neuropsychol. Rev. 24, 3-15. doi: 10.1007/s11065-014-9251-Z

Raichle, M. E., MacLeod, A. M., Snyder, A. Z., Powers, W. J., Gusnard, D. A., and Shulman, G. L. (2001). A default mode of brain function. Proc. Natl. Acad. Sci. U.S.A. 98, 676-682. doi: 10.1073/pnas.98.2.676

Richman, J. S., and Moorman, J. R. (2000). Physiological time-series analysis using approximate entropy and sample entropy. Am. J. Physiol. Heart Circ. Physiol. 278, H2039-H2049.

Saad, Z. S., Gotts, S. J., Murphy, K., Chen, G., Jo, H. J., Martin, A., et al. (2012). Trouble at rest: how correlation patterns and group differences become distorted after global signal regression. Brain Connect. 2, 25-32. doi 10.1089/brain.2012.0080

Satterthwaite, T. D., Elliott, M. A., Gerraty, R. T., Ruparel, K., Loughead, J., Calkins, M. E., et al. (2013). An improved framework for confound regression and filtering for control of motion artifact in the preprocessing of resting-state functional connectivity data. Neuroimage 64, 240-256. doi: 10.1016/j.neuroimage.2012.08.052

Seeley, W. W., Crawford, R. K., Zhou, J., Miller, B. L., and Greicius, M. D. (2009). Neurodegenerative diseases target large-scale human brain networks. Neuron 62, 42-52. doi: 10.1016/j.neuron.2009.03.024

Seeley, W. W., Menon, V., Schatzberg, A. F., Keller, J., Glover, G. H., Kenna, H., et al. (2007). Dissociable intrinsic connectivity networks for salience processing and executive control. J. Neurosci. 27, 2349-2356. doi: 10.1523/JNEUROSCI.558706.2007

Shew, W. L., Yang, H., Petermann, T., Roy, R., and Plenz, D. (2009). Neuronal avalanches imply maximum dynamic range in cortical networks at criticality. J. Neurosci. 29, 15595-15600. doi: 10.1523/JNEUROSCI.3864-0 9.2009

Shew, W. L., Yang, H., Yu, S., Roy, R., and Plenz, D. (2011). Information capacity and trans- mission are maximized in balanced cortical networks with neuronal avalanches. J. Neurosci. 31, 55-63. doi: 10.1523/JNEUROSCI.463710.2011

Shulman, G. L., Fiez, J. A., Corbetta, M., Buckner, R. L., Miezin, F. M., Raichle, M. E., et al. (1997). Common blood flow changes across visual tasks: II. Decreases in cerebral cortex. J. Cogn. Neurosci. 9, 648-663. doi: 10.1162/jocn.1997. 9.5.648

Small, G. W., Ercoli, L. M., Silverman, D. H., Huang, S. C., Komo, S., Bookheimer, S. Y., et al. (2000). Cerebral metabolic and cognitive decline in persons at genetic risk for Alzheimer's disease. Proc. Natl. Acad. Sci. U.S.A. 97, 6037-6042. doi: 10.1073/pnas.090106797 
Smith, R. X., Yan, L., and Wang, D. J. J. (2013). Multiple time scale complexity analysis of resting state FMRI. Brain Imaging Behav. 8, 284-291. doi: 10.1007/ s11682-013-9276-6

Smith, S. M., Fox, P. T., Miller, K. L., Glahn, D. C., Fox, P. M., Mackay, C. E., et al. (2009). Correspondence of the brain's functional architecture during activation and rest. Proc. Natl. Acad. Sci. U.S.A. 106, 13040-13045. doi: 10.1073/pnas.0905267106

Sokunbi, M., Fung, W., Sawlani, V., Choppin, S., Linden, D., and Thome, J. (2013). Resting state fMRI entropy probes complexity of brain activity in adults with ADHD. Psychiatry Res. 214, 341-348. doi: 10.1016/j.pscychresns.2013.10.001

Sokunbi, M. O., Staff, R. T., Waiter, G. D., Ahearn, T. S., Fox, H. C., Deary, I. J., et al. (2011). Inter-individual differences in fMRI entropy measurements in old age. IEEE Trans. Biomed. Eng. 58, 3206-3214. doi: 10.1109/TBME.2011.2164793

Song, X.-W., Dong, Z. Y., Long, X. Y., Li, S.-F., Zuo, X.-N., Zhu, C.-Z., et al. (2011). REST: a toolkit for resting-state functional magnetic resonance imaging data processing. PLoS ONE 6:e25031. doi: 10.1371/journal.pone.0025031

Spreng, R. N., Mar, R. A., and Kim, A. S. N. (2009). The common neural Basis of autobiographical memory, prospection, navigation, theory of mind, and the default mode: a quantitative metaanalysis. J. Cogn. Neurosci. 21, 489-510. doi: 10.1162/jocn.2008.21029

Takahashi, T., Cho, R. Y., Mizuno, T., Kikuchi, M., Murata, T., Takahashi, K., et al. (2010). Antipsychotics reverse abnormal EEG complexity in drug-naive schizophrenia: a multiscale entropy analysis. Neuroimage 51, 173-182. doi: 10.1016/j.neuroimage.2010.02.009

Tononi, G., Edelman, G. M., and Sporns, O. (1998). Complexity and coherency: integrating information in the brain. Trends Cogn. Sci. 2, 474-484. doi: 10.1016/S1364-6613(98)01259-5

Tononi, G., Sporns, O., and Edelman, G. M. (1994). A measure for brain complexity: relating functional segregation and integration in the nervous system. Proc. Natl. Acad. Sci. U.S.A. 91, 5033-5037. doi: 10.1073/pnas.91.11.5033

Touroutoglou, A., Hollenbeck, M., Dickerson, B. C., and Barrett, L. F. (2012). Dissociable large-scale networks anchored in the right anterior insula subserve affective experience and attention. Neuroimage 60, 1947-1958. doi: 10.1016/j.neuroimage.2012.02.012

Vakorin, V. A., Lippé, S., and McIntosh, A. R. (2011). Variability of brain signals processed locally transforms into higher connectivity with brain development. J. Neurosci. 31, 6405-6413. doi: 10.1523/JNEUROSCI.3153-10.2011

van den Heuvel, M. P., Stam, C. J., Kahn, R. S., and Hulshoff Pol, H. E. (2009). Efficiency of functional brain networks and intellectual performance. J. Neurosci. 29, 7619-7624. doi: 10.1523/JNEUROSCI.1443-09.2009

Van Essen, D. C., Dickson, J., Harwell, J., Hanlon, D., Anderson, C. H., and Drury, H. A. (2001). An integrated software system for surface-based analyses of cerebral cortex. J. Am. Med. Inform. Assoc. 8, 443-459. doi: 10.1136/jamia.2001.0080443

Van Essen, D. C., Smith, S. M., Barch, D. M., Behrens, T. E. J., Yacoub, E., and Ugurbil, K. (2013). The WU-Minn Human Connectome Project: an overview. Neuroimage 80, 62-79. doi: 10.1016/j.neuroimage.2013.05.041 van Leeuwen, C. (2008). Chaos breeds autonomy: connectionist design between bias and baby-sitting. Cogn. Process. 9, 83-92. doi: 10.1007/s10339-007-0193-8

Vincent, J. L., Kahn, I., Snyder, A. Z., Raichle, M. E., and Buckner, R. L. (2008). Evidence for a frontoparietal control system revealed by intrinsic functional connectivity. J. Neurophysiol. 100, 3328-3342. doi: 10.1152/jn.90355.2008

Wiesenfeld, K., and Moss, F. (1995). Stochastic resonance and the benefits of noise: from ice ages to crayfish and SQUIDs. Nature 373, 33-36. doi: 10.1038/373033a0

Wig, G. S., Schlaggar, B. L., and Petersen, S. E. (2011). Concepts and principles in the analysis of brain networks. Ann. N. Y. Acad. Sci. 1224, 126-146. doi: 10.1111/j.1749-6632.2010.05947.x

Yang, A. C., Huang, C.-C., Liu, M.-E., Liou, Y.-J., Hong, C.-J., Lo, M.-T., et al. (2013a). The APOE ?4 allele affects complexity and functional connectivity of resting brain activity in healthy adults. Hum. Brain Mapp. doi: 10.1002/hbm. 22398. [Epub ahead of print].

Yang, A. C., Huang, C.-C., Yeh, H.-L., Liu, M.-E., Hong, C.-J., Tu, P.-C., et al. (2012). Complexity of spontaneous BOLD activity in default mode network is correlated with cognitive function in normal male elderly: a multiscale entropy analysis. Neurobiol. Aging 34, 428-438. doi: 10.1016/j.neurobiolaging.2012. 05.004

Yang, A. C., and Tsai, S.-J. (2013). Is mental illness complex? From behavior to brain. Prog. Neuro-Psychopharmacol. Biol. Psychiatry 45, 253-257. doi: 10.1016/j.pnpbp.2012.09.015

Yang, A. C., Wang, S.-J., Lai, K.-L., Tsai, C.-F., Yang, C.-H., Hwang, J.-P., et al. (2013b). Cognitive and neuropsychiatric correlates of EEG dynamic complexity in patients with Alzheimer's disease. Prog. Neuropsychopharmacol. Biol. Psychiatry 47, 52-61. doi: 10.1016/j.pnpbp.2013.07.022

Zarahn, E., Aguirre, G. K., and D’Esposito, M. (1997). Empirical analyses of BOLD fMRI statistics. I. Spatially unsmoothed data collected under null-hypothesis conditions. Neuroimage 5, 179-197.

Conflict of Interest Statement: The authors declare that the research was conducted in the absence of any commercial or financial relationships that could be construed as a potential conflict of interest.

Received: 10 March 2014; paper pending published: 07 April 2014; accepted: 22 May 2014; published online: 10 June 2014.

Citation: McDonough IM and Nashiro K (2014) Network complexity as a measure of information processing across resting-state networks: evidence from the Human Connectome Project. Front. Hum. Neurosci. 8:409. doi: 10.3389/fnhum.2014.00409 This article was submitted to the journal Frontiers in Human Neuroscience. Copyright (c) 2014 McDonough and Nashiro. This is an open-access article distributed under the terms of the Creative Commons Attribution License (CC BY). The use, distribution or reproduction in other forums is permitted, provided the original author(s) or licensor are credited and that the original publication in this journal is cited, in accordance with accepted academic practice. No use, distribution or reproduction is permitted which does not comply with these terms. 